\title{
Interplay of seismic and aseismic deformations during earthquake swarms: an experimental approach
}

\author{
O. Lenglinéa,*, J. E. Elkhoury ${ }^{b}$, G. Daniel ${ }^{c}$, J. Schmittbuhl $^{\mathrm{a}}$, R. Toussaint ${ }^{\mathrm{a}}$, \\ J.-P. Ampuero ${ }^{\mathrm{b}}$, M. Bouchon ${ }^{\mathrm{d}}$ \\ ${ }^{a}$ Institut de Physique du Globe de Strasbourg, CNRS et Université de Strasbourg \\ (EOST), France \\ ${ }^{b}$ Seismological Laboratory, Caltech, Pasadena, USA \\ ${ }^{c}$ Magnitude, Research and Development, Centre Regain, Sainte-Tulle, France \\ ${ }^{d}$ ISTERRE, CNRS et Université Joseph Fourier, Grenoble, France
}

\begin{abstract}
Observations of earthquake swarms and slow propagating ruptures on related faults suggest a close relation between the two phenomena. Earthquakes are the signature of fast unstable ruptures initiated on localized asperities while slow aseismic deformations are experienced on large stable segments of the fault plane. The spatial proximity and the temporal coincidence of both fault mechanical responses highlight the variability of fault rheology. However, the mechanism relating earthquakes and aseismic processes is still elusive due to the difficulty of imaging these phenomena of large spatiotemporal variability at depth. Here we present laboratory experiments that explore, in great detail, the deformation processes of heterogeneous interfaces in the brittle-creep regime. We track the evolution of an interfacial crack over 7 orders of magnitude in time and 5 orders of magnitude in space using optical and acoustic sensors. We explore the response of the system to slow transient loads and show that slow deformation episodes are systematically accompanied by acoustic emissions due to local fracture energy disorder. Features of acoustic emission activities and deformation rates distributions of our experimental system are similar to those in natural faults. On the basis of an activation energy model, we link our results to the Rate and State friction model and suggest an active role of local creep deformation in driving the
\end{abstract}

\footnotetext{
${ }^{*}$ Corresponding author

Email address: lenglineunistra.fr (O. Lengliné)
} 
seismic activity of earthquake swarms.

Keywords:

\section{Introduction}

Numerous observations of a correlation between seismic activity enhancements and slow slip transients in the Earth crust have been reported in various tectonic locations (Linde et al., 1996; Crescentini et al., 1999; Lohman and McGuire, 2007; Segall et al., 2006; Liu et al., 2007) and in geothermal areas (Bourouis and Bernard, 2007; Takada and Furuya, 2010). Also, observations of postseismic slip and aftershocks following large earthquakes are manifestations of transient deformation coupled with abundant earthquake activity (Perfettini and Avouac, 2004). A central question is whether or not these two different mechanical responses of faults, i.e. seismic and aseismic slip, occur on closely located zones. If they are, one expects a strong interaction between the two processes.

In some cases, the seismic signal concurrent with slow slip events is characterized as tectonic tremors or low frequency earthquakes, as observed in subduction zones (Rogers and Dragert, 2003; Obara et al., 2004; Ito et al., 2007) or in transform tectonic settings like the San Andreas Fault (SAF) (Vidale and Shearer, 2006; Nadeau and Dolenc, 2005; Shelly, 2010). The aseismic nucleation phase of mainshocks has also been related to foreshocks, as in the case of the 1999 Izmit earthquake (Bouchon et al., 2011) or the 2011 Tohoku-Oki earthquake (Miyazaki et al., 2011; Ando and Imanishi, 2011; Kato et al., 2012). Seismic events associated with aseismic slip are generally located on fault planes suggesting that they represent a dynamic shear instability on the sliding interface (La Rocca et al., 2009; Shelly et al., 2009). Geodetic inversions suggest that the aseismic motion occurs on the same fault plane as the seismic events (Lohman and McGuire, 2007). However the deformation signal recorded at the surface by GPS and/or InSAR instruments only provides a macroscopic view of the deformation process at depth. Details of the aseismic slip distribution are often lacking due to the limited resolution, unless the aseismic slip occurs at the Earth surface (Doubre and Peltzer, 2007).

The close spatial and temporal occurrence of both seismic and aseismic slip suggests a causal relation between the two phenomena. Nonetheless, the causal mechanism is not straightforward as earthquakes can both trigger 
and be triggered by slow slip events (Du et al., 2003; Perfettini and Avouac, 2004). It might also be that both aseismic and seismic slip are manifestations of a common deformation process. In this case, the seismic signal can be seen as the signature of patches on the fault plane deforming dynamically whereas the geodetic observation represents an integrated signal over all the deforming sites. Therefore, the deformation on a fault plane takes place over a wide range of speeds. This is supported by observations of heterogeneous postseismic slip on the SAF inferred from the analysis of repeating earthquake sequences (Lengliné and Marsan, 2009). The dynamic events represent the high velocity tail of the slip speed distribution while its average produces the observed geodetic signal.

The contribution of earthquakes to the total amount of slip released during transient episodes is generally small (Lohman and McGuire, 2007). Despite being located on a common interface, accurate spatial location of the seismic activity relative to the aseismic slip is difficult to obtain. The heterogeneity of mechanical and physical properties of the interface might control the partition between seismic and aseismic slip, the interface being envisioned as a collection of brittle patches (asperities) embedded in an otherwise creeping region which accommodates slow slip (Linde et al., 1996; Lohman and McGuire, 2007; Wech et al., 2009; Perfettini et al., 2010). The concentration of asperities, or brittle patches, governs the relative importance of dynamic failures in the deformation process. It is also readily possible that temperature produces a broad scale effect on the slip partition over the interface and thus constrains the transition between brittle and ductile rheology.

The physics of both seismic and aseismic processes is not straightforward as it involves a complex problem on a spatially heterogeneous medium with a large number of degrees of freedom and short and long range interactions. Several numerical models have tried to reproduce the evolution of such systems but computations are extremely demanding and time consuming and limited to only large scale heterogeneities (Kaneko et al., 2010), or to the quasi-dynamic approximation (Hillers et al., 2007; Ariyoshi et al., 2011). Analog laboratory experiments overcome these difficulties as space-time integrations are performed by the deforming system without any model requirement. Here, we investigate the slow (creep) and fast (acoustic) deformations produced by the propagation of an interfacial brittle-creep crack. Our experimental setup is a much simpler configuration than that of a complex fault zone system but the fundamental processes of interest, the interplay of slow deformation and brittle fracture on the same heterogeneous interface, remain 
similar. Our setup allows the simultaneous monitoring of both acoustic activity and the detailed geometrical evolution of the fracture. We show that seismic and aseismic events co-exist in the system and their activity rates are highly correlated. This suggests that seismic activity during swarm episodes is driven by the elastic loading of asperities by local aseismic deformations.

\section{Experimental Setup}

\subsection{Sample Preparation}

To prepare each sample, we use two transparent PMMA (poly methyl methacrylate) plates of dimensions $20 \times 10 \times 1.0 \mathrm{~cm}$ and $23 \times 2.8 \times 0.5 \mathrm{~cm}$ (Figure 1). First, we sand-blast one surface of the narrow plate with glass beads of diameter $\phi \in[180-300] \mu \mathrm{m}$. We clean the blasted plate to remove any electrically attached glass beads. Then we assemble the two plates in a stiff metallic loading cell with the blasted surface facing one of the surfaces of the thick plate. Finally, we impose a homogeneous normal load on the assembled plates and heat the loaded sample to $190^{\circ} \mathrm{C}$ for 45 minutes to anneal the plates. The thermal annealing produces a cohesive interface, weaker than the bulk, along which the sample will break under load. The sand-blasting introduces random roughness to the plate surface that controls the heterogeneity of local strength along the interface. It also induces microstructures on the plate surface that make the surface opaque. The newly formed block, after annealing, recovers its transparency since the contrast of the refraction index along the interface disappears (see Grob et al. (2009); Lengliné et al. (2011) for details). Interestingly PMMA exhibits a brittle behavior at short time scales and is semi-brittle or even plastic at longer times. Macroscopically this long time scale regime is described by a ductile rheology. PMMA shows a time-temperature equivalence desirable for addressing either high temperature processes or very long term evolution (Ward and Hadley, 1993). This richness of the PMMA rheology enables the observation of a brittle-creep rupture regime. It provides an attractive analogy for the study of numerous time-dependent mechanisms in the Earth crust as those originating at the brittle-ductile transition.

\subsection{Mechanical loading}

Once the sample is ready, we clamp the widest PMMA plate to a stiff aluminum frame. A stepping motor applies the loading over the top side of the narrow plate in a direction normal to the plate interface (Figure 1). 
We measure the vertical displacement of the loading point with a linear variable differential transformer (LVDT) with a resolution of $1.3 \mu \mathrm{m}$. The vertical displacement imposed on the narrower plate induces stable mode I propagation of a planar fracture along the prescribed weak interface. We impose a variation of the loading speed, $\dot{u}(t)$, to simulate a variation of the external driving force. We performed 14 experiments where we applied various forms of temporal transients, namely a step increase, a bump increase and a sinusoidal of the loading speed imposed by the stepping motor. All these transient loads produced broad scale variation of the front speed (e.g. Figure 2, see also Supplementary Material) and they could be interpreted as an analog for far field stress perturbations caused, for example, by pore fluid pressure, magmatic intrusion or mantle flow.

\subsection{Optical events}

We monitor the fracture front propagation using a fast camera (CamRecord 600) with up to 1000 fps or a slow speed camera (Nikon D700) with up to 5 fps to follow the progression of the front position over longer time scales (Figure 1). Optical images of the interfacial rupture show dark and bright regions corresponding to open crack and unbroken parts of the sample respectively. Image processing determines the transition between dark and bright areas that defines the fracture front. We first compute the difference between each image and the first image of the experiment. The image difference highlights the fracture front while removing permanent artifacts. Then, grayscale images are transformed into black and white images according to a gray level threshold separating bright and dark regions. Then, we calculate the gradient in the direction of front propagation to highlight the transition zone. We finally extract connected pixels from the gradient images that correspond to the front position, $a(x, t)$. The front propagates along the $y$ axis with the origin defined at the loading point and is positive in the direction of crack propagation. The $x$ axis is perpendicular to $y$ and defines the coordinate of a point along the front and $\bar{a}(t)$ is the mean position of the front at time $t$ (see Måløy et al. (2006) and Grob et al. (2009) for details). We compute the local speed of the crack as the pixel size divided by the time spent by the front in each pixel (Figure 3). We extract the pixels with the highest speeds from the local movements of the front. The broad scale variation of the front velocity could be compared to slow events recorded by geodetic instruments in a tectonic context. The patches of higher than average deformation speed could be considered as analogous to local creeping 
episodes or slow slip events. They represent slow deformation episodes that locally exceed the macroscopic deformation rate. We follow the procedure detailed by Grob et al. (2009) to define such events. We define an "optical event" (OE) as a as a cluster of connected pixels which velocity exceeds a prescribed speed threshold, $v_{t h}$. Here we adopt $v_{t h}=10 \times\langle v\rangle$, where $\langle v\rangle$ is the mean velocity of the crack during an experiment. Although these optical events have higher velocities than most of the deforming sites, they remain slow deformation episodes compared to dynamic events (the average speed $\langle v\rangle \simeq 500 \mu \mathrm{ms}^{-1}$, far from the Rayleigh wave speed, $\left.V_{r} \simeq 1.710^{9} \mu \mathrm{ms}^{-1}\right)$. These optical events are characterized by a Gutenberg-Richter power law with a slope similar to that of tectonic earthquakes (Grob et al., 2009). This Gutenberg-Richter relation might also be related to the magnitude-frequency scaling inferred for slow-slip events (Wech et al., 2010).

\subsection{Acoustic events}

The crack propagation produces acoustic activity that we monitor with a 32 elements linear array of piezo-electric sensors. Sensors are located on a line parallel to the plate axis and sensors separation is $3 \mathrm{~mm}$ (see Fig. 1). The typical distance between the closest acoustic sensor and the border of the plate is $1 \mathrm{~cm}$. The sensors peak frequency response is $\sim 500 \mathrm{kHz}$ and all channels are continuously recorded at $5 \mathrm{MHz}$. For each experiment, we extract the acoustic signal recorded in the two closest acoustic sensors to the fracture front line. We manually trigger the acquisition of the acoustic data, which is synchronized with the camera time sequence. The recordings of the acoustic signals last, in our experiments, for a maximum of $50 \mathrm{~s}$.

We use a short-term-average to long-term-average ratio (STA/LTA) to detect acoustic events (AE) in the recorded acoustic signal (Earle and Shearer, 1994). This procedure is similar to that applied to earthquake data. In our case, we use shorter time windows tailored to our short signal durations and high frequency acquisition rate. The STA window length is $20 \mu s$, the LTA window length is $100 \mu \mathrm{s}$ and the threshold for setting an STA/LTA detection is 2.0. An event must be detected simultaneously on the two closest channels to be considered in our analysis. We show an example of the acoustic signal recorded during one experiment in Figure 4. The typical duration of the recorded events is on the order of $100 \mu \mathrm{s}$. The performance of the detection algorithm is illustrated in Figure 5. As expected, the detection of events is associated with high amplitudes of the recorded signal on the two closest channels. AEs result from the fracturing of geometrical asperities over the 
interface. We estimate the typical spatial scale of these failures to be less than several microns (The largest AE is generated during an optical event that has maximum dimensions of $\sim 600 \mu \mathrm{m}$ by $30 \mu \mathrm{m})$. We observe nonuniformly distributed clustering of acoustic and optical activity (Figure 5). The temporal coincidence of AEs and OEs is important but not systematic (Figure 5). It is attributed to AEs that are too small to be optically detected, OEs that are genuinely aseismic, and clusters of AEs that are lumped into a single large OE.

\section{Results}

\subsection{Distribution of inter-event time}

Our results suggest the presence of clustering of the acoustic activity (Figure 5). We quantify this clustering by calculating the distribution of inter-event times of successive AEs for all 14 experiments. We only considered AE when the loading rate was nearly constant in order to avoid mixing populations recorded during different loading rates. Inter-event times are normalized by the average AE rate of each experiment (the average AE rate for the different experiments, is on the order of $10^{2}$ events/sec). The probability density functions (pdf) for all the experiments are well approximated by a gamma function (Figure 6). The displayed gamma distribution is obtained from averaging the parameters of each individual fit. Figure 6 also includes the inter-event time distribution for the ISC worldwide catalog for earthquakes with magnitude $\mathrm{M}>5.5$ in the period 1975-2004. Temporal clustering has been well documented for earthquakes and is suggested to be a result of interactions among earthquakes (Corral, 2004; Molchan, 2005; Hainzl et al., 2006; Saichev and Sornette, 2007). The normalization of the interevent time is obtained similarly by the average seismic event rate (on the order of 1 event/day). The good agreement between the gamma distribution and the pdf obtained from our experimental data is similar to that for earthquake data (Corral, 2004) or AE recorded during rock fracture experiments (Davidsen et al., 2007). It suggests that the mechanism responsible for the seismic activity in fault systems or rock fractures shares strong similarities with our experiment, which can be seen as an analogous system for seismicity interactions. We interpret the decay at short time scale as the emergence of an Omori-Utsu law describing interactions among events while the distribution evolves to an exponential distribution representative of a Poisson process at longer time scales (Saichev and Sornette, 2007). 


\subsection{Power-law distribution of local slip-rate}

We calculate the pdf of the local velocities following Måløy et al. (2006) for six of the experiments where we used the fast video camera at high sampling rate. We only analyze, for these experiments, time windows with an almost constant large scale velocity. The local velocity is normalized by the average velocity of the crack, $\langle v\rangle$. Front velocities exhibit significant fluctuations at small scales (Figure 3). For $v>\langle v\rangle$ the pdf shows a power law decay with exponent 2.55 consistent with previous works (Måløy et al., 2006; Lengliné et al., 2011). This power law behavior of the deformation rate at small scale, in our experiment, has to be compared to the observed behavior in natural fault systems. For instance, in southern California, fault slip rates are found to obey such a power law scaling where the fault system is characterized by slow slip rates (Meade, 2007). This comparison suggests that a second relation between our experiments and natural fault behavior can be proposed and that the scaling behavior is a general feature of slowly deforming media in the presence of heterogeneities.

\subsection{Lateral migration of the deformation velocity}

Migration of the fracture velocity along the crack front direction is analogous to the dip-parallel slip propagation revealed by tremors in subduction zone (Shelly et al., 2007; Ghosh et al., 2010). In most experiments, high loading rates or low recording acquisition rates do not allow us to distinguish migration patterns confidently as interactions among sites are numerous and occur over short time intervals. We find indications of along-front migration with tractable velocities for long enough times during experiments where loading rates are the lowest (Figure 8). High velocity clusters appear in streaks that suggest the progression of the rupture in the direction perpendicular to the crack front propagation. The migration velocity of these high velocity streaks is on the order of $2 \mathrm{~cm} / \mathrm{s}$ (Figure 8). During this time interval, the crack front propagated at a typical velocity of $400 \mu \mathrm{m} / \mathrm{s}$. We note that this migration speed is higher than the crack front speed. Taking 10-100 $\mathrm{km} / \mathrm{h}$ as a typical range of dip-parallel tremor migration (Shelly et al., 2007; Ghosh et al., 2010), and $10 \mathrm{~km} /$ day as an estimate of along-strike speed of the slow slip event (e.g. Bartlow et al., 2011), the ratio of these two velocities is in the range 25-250. The velocity ratio obtained in our experiment is on the order of 50, well within the range obtained in subduction zones. 


\subsection{An interplay of seismic and aseismic local deformations}

The evolution of the acoustic emission (AE) rate, the spatial average velocity of the crack front and the optical event (OE) rate are similar (Figure 7). $\mathrm{AE}, \mathrm{OE}$ rates and the average front velocities are computed for intervals of $0.2 \mathrm{~s}$. A clear temporal relation between these three quantities at this large scale can be inferred: the AE and OE rates closely follow the macroscopic variation of crack front speed. The brittle fracture propagation generating acoustic emission and creep deformation co-exist in the same zone and are active at the same time.

We calculate the affine relation connecting the average front speed and the AE rate for all experiments. The residuals of the linear relation are small and symmetrically distributed suggesting a linear relation between AE rate and crack velocity. This is attested by the correlation coefficient, $\rho$, computed for each experiment between these two variables. The correlation coefficient is defined as $\rho=\sigma_{x y} / \sigma_{x} \sigma_{y}$, with $\sigma_{x y}$ the covariance of the AE rate with the front speed and $\sigma_{x}$ and $\sigma_{y}$ the standard deviations of the AE rate and the front speed. The correlation coefficient, $\rho$ for a $0.2 \mathrm{~s}$ window and for our 14 experiments ranges from $\rho=0.75$ to 0.97 and with a mean value of 0.87 .

We now focus on smaller time scales relevant to acoustic activity and slow movement of the crack front. The crack front velocity shows important fluctuations at small scales, although being smooth and rather continuous when observed at large scale. These fluctuations result from the heterogeneous nature of the interface and the elastic interactions along the crack front line. We investigate the temporal relation between $\mathrm{OE}$ and $\mathrm{AE}$ recorded during the 6 experiments which show the highest acoustic activity and the best resolved crack advance. The cross-correlation function, $C(\Delta t)$, between the rate of $\mathrm{AE}, r_{A E}(t)$, and the rate of optical events, $r_{O E}(t)$ is

$$
C(\Delta t)=\frac{1}{\sqrt{C_{A E} C_{O E}}} \sum_{t_{0}}^{t_{f}} r_{O E}(t+\Delta t) \times r_{A E}(t),
$$

where $C_{A E}$ and $C_{O E}$ are the auto-correlations values at zero lag time of the $\mathrm{AE}$ and the $\mathrm{OE}$ rates, respectively. For both $\mathrm{AE}$ and $\mathrm{OE}$, rates are computed as the number of events detected in time intervals of $5 \mathrm{~ms}$ from time $t_{0}$ to time $t_{f}$ (the mean is removed from the time-series). The maximum correlation between the two types of signals is at zero lag time (Figure 9). It shows that the acoustic activity occurs mostly in a short time span around the aseismic deformation. The quasi-symmetric shape of the correlation function also 
suggests that optical events both precede and follow acoustic activity during the course of an experiment highlighting the close interplay between these two modes of deformation. The slight asymmetrical shape of $C(\Delta t)$ (Figure 9) also suggests that a higher rate of $\mathrm{OE}$ occurs following AE than preceding it, similar to postseismic slip observed after large earthquakes (e.g. Hsu et al., 2006).

\section{Discussion}

In our experiments, the average fracture speed obeys the Arrhenius law at the macroscopic or global scale (Lengliné et al., 2011). Such an evolution has been inferred in numerous experimental systems reproducing slow deformations of rocks and other materials (Atkinson, 1984). In our experiment, this expression relates the crack front speed propagation, $V$, to an activation energy mechanism and can be expressed as

$$
V=V_{0} \exp \left(\frac{-Q}{k_{B} T}\right)
$$

$V_{0}$ is the product of a frequency of attempt to break molecular bonds and the jump distance, $k_{B}$ is the Boltzmann constant and $T$ is the temperature. The activation energy of the process is $Q$

$$
Q=Q_{0}-G \alpha^{2}
$$

where $Q_{0}$ is the activation energy in the absence of applied stress, $G$ is the energy release rate and $\alpha$ is the typical length scale of the atomistic fracturing process. The stress dependence in Eq. (3) is similarly often written in terms of the stress intensity factor, $K$, at the crack tip. It follows that by controlling the imposed loading rate on the system we modify the value of the energy release rate (equivalently stress intensity factor) at the crack tip. In this sense, simulated transient deformation processes mimic the increase of the deformation speed recorded during swarm episodes (Lohman and McGuire, 2007) or slow slip events in subduction zones (Rogers and Dragert, 2003). The formulation of the crack speed in Eq. (2) has been proposed to be the physical basis of the rate and state friction model widely considered in fault mechanics (e.g. Nakatani, 2001; Rice et al., 2001). If we consider a volumetric process, the corresponding expression to Eq. (3) is

$$
Q=Q_{0}-\tau_{c} \alpha^{3}
$$


where the stress dependence is written in terms of the average shear stress, $\tau_{c}$ along asperity contacts. $\alpha^{3}$ is the activation volume of the process. Following Rice et al. (2001), we call $\sigma_{c}$ the average normal stress on contact zones, defined as: $\tau_{c} / \sigma_{c}=\tau / \sigma$, with $\tau$ and $\sigma$ being the large scale stresses. It follows from Eq. (2)

$$
V=V_{0} \exp \left(\frac{-Q_{0}+\tau_{c} \alpha^{3}}{k_{B} T}\right)
$$

leading to:

$$
\tau=\sigma\left[\frac{Q_{0}}{\alpha^{3} \sigma_{c}}+\frac{k_{B} T}{\sigma_{c} \alpha^{3}} \ln \left(\frac{V}{V_{0}}\right)\right]
$$

where $V$ is interpreted in terms of slip rate on the fault plane. Eq. 6 has a similar form to the empirical relation of the rate and state friction model with $a=k_{B} T / \sigma_{c} \alpha^{3}$ and the state dependent variable represented by $Q_{0} / \alpha^{3} \sigma_{c}$ (Rice et al., 2001). It follows that the progressive evolution of the front position in our system can be viewed as a similar mechanical problem to the evolution of slip on a fault plane with the same constitutive equation. The quasi-static evolution of an anti-plane shear rupture is governed by similar formulations to mode I fractures (Gao and Rice, 1986; Schmittbuhl et al., 2003). Thus, we consider our mode I fracture problem analogous to shear ruptures. Therefore our experimental setup is relevant for addressing the mechanics of simplified fault models as studied numerically by Gao et al. (1991); Perfettini et al. (2003). Provided this analogy, the crack front advance $a(x, t)$ is analogous to the slip along fault $\delta(x, t)$. However each point of the interface that breaks is completely unloaded due to the geometry of our experimental setting. Therefore, we have no repeating failure of the same patch contrary to natural faults and AE stress drops are total. However, earthquake stress drops are usually a small fraction of the total stress (e.g. Kanamori, 1994). Nevertheless, in both systems, AE and earthquake still represent a dynamic failure mode, which makes them comparable. Following the approach presented by Dieterich (1994), we propose to relate the AE rate to the stress history of our system. We assume an interface composed of a population of sources that generate AE. We hypothesize that the duration of nucleation of these sources is longer than the duration of stress variations imposed to the system. Hence, the seismicity rate, $R$, varies exponentially with the shear stress change $\Delta \tau$ imposed on the system (Dieterich, 1994; 
Beeler and Lockner, 2003; Cochran et al., 2004; Helmstetter and Shaw, 2009),

$$
R=r \exp \left(\frac{\Delta \tau}{a \sigma}\right)
$$

where $r$ is a reference seismicity rate. The analogy mentioned above between shear stress $\tau$ and the energy release rate $G$, suggests that in our experiment:

$$
R=r \exp \left(\frac{\alpha^{2} \Delta G}{k_{B} T}\right),
$$

Notice that Eq. (8) is the same expression of that for the speed of the crack as a function of $G$ obtained by combining Eq. (2) and (3): $V=$ $V_{0}^{*} \exp \left(\alpha^{2} G / k_{B} T\right)$ with $V_{0}^{*}=V_{0} \exp \left(-Q_{0} / k_{B} T\right)$. Therefore we have a direct linear relation between $\mathrm{AE}$ rate $R$ and speed of the crack $V: R \propto V$, (Figure 7). At the system size, this relation provides a link between acoustic activity and periods of high deformation. At smaller scales, the same mechanism is applicable as the acoustic activity is influenced by local important deformations episodes (OE) (Figure 9). Such local creeping episodes are similar to the small localized creep events observed on the San-Andreas fault by Scholz et al. (1969).

We emphasize that the deformation recorded at the surface of the Earth during transient episodes should be viewed as the macroscopic integration of sequences of creep events. On large spatial scales, the creep events formed the observed large scale behavior and correspond to the macroscopic observation of the transient slip speed variation. Such a view is similar to the concept developed by Scholz et al. (1969) where the deformation is modeled as the integration of discrete sites subject to time-dependent strength. The acoustic deformation being recorded only represents the part of the deformation corresponding to zones that fail dynamically. This model is fully compatible with our observations. We also remark that sites do not fail independently as attested by the local close temporal activity between creep events and acoustic events. As proposed by Scholz et al. (1969), it is readily possible that creep events recorded at our experimental scale are also formed of small acoustic events such that down-scaling might exist up to the microscopical level associated with the discrete breakage of single molecular bonds.

\section{Conclusion}

We analyze the coupled evolution of acoustic activity and slow deformation during the propagation of brittle-creep fractures in a heterogeneous 
medium. Our unique experimental setup addresses the relation between seismic and aseismic slip along natural faults. It provides an original characterization of slow deformation processes which are difficult to capture on faults at depth. Numerous statistical features of the deformation observed in natural systems are reproduced by our experiments like the gamma distribution of interevent times and the power law distribution of slip rates. We show that the acoustic activity (dynamic events) is part of the deformation process and it occurs over a widely distributed range of speeds including slow slip. Also, small scale observations of the deformation reveal coexistence between creep and acoustic events in the same mechanical system. The small scale complex dynamics lead to a macroscopic integrated signal of the deformation that shows a smooth and continuous deformation speed that correlates with the rate of acoustic events.

\section{Acknowledgments}

We thank K. J. Måløy, K. T. Tallakstad, S. Santucci, M. Grob, F. H. Cornet, J.P. Avouac, D. R. Shelly, M. Aktar and H. Karabulut for fruitful discussions. We also thank the editor and two anonymous reviewers for their suggestions and A. Steyer for technical support. We acknowledge the support of ANR grant SUPNAF and of NSF grant EAR-1015698.

\section{References}

Ando, R., Imanishi, K., 2011. Possibility of Mw 9.0 mainshock triggered by diffusional propagation of after-slip from mw 7.3 foreshock. Earth, Planets and Space 63, 767-771.

Ariyoshi, K., Matsuzawa, T., Ampuero, J.-P., Nakata, R., Hori, T., Kaneda, Y., Hino, R., Hasegawa, A., 2011. Migration process of very low-frequency events based on a chain-reaction model and its application to the detection of preseismic slip for megathrust earthquakes. Earth, Planets and Space, in press.

Atkinson, B. K., Jun. 1984. Subcritical crack growth in geological materials. J. Geophys. Res. 89, 4077-4114.

Bartlow, N. M., Miyazaki, S., Bradley, A. M., Segall, P., 2011. Space-time correlation of slip and tremor during the 2009 Cascadia slow slip event. Geophys. Res. Lett. 38, 18309. 
Beeler, N. M., Lockner, D. A., 2003. Why earthquakes correlate weakly with the solid Earth tides: Effects of periodic stress on the rate and probability of earthquake occurrence. J. Geophys. Res. 108, 2391.

Bouchon, M., Karabulut, H., Aktar, M., Özalaybey, S., Schmittbuhl, J., Bouin, M.-P., 2011. Extended Nucleation of the $1999 M_{w} 7.6$ Izmit Earthquake. Science 331, 877.

Bourouis, S., Bernard, P., 2007. Evidence for coupled seismic and aseismic fault slip during water injection in the geothermal site of Soultz (France), and implications for seismogenic transients. Geophys. J. Int. 169, 723-732.

Cochran, E. S., Vidale, J. E., Tanaka, S., 2004. Earth Tides Can Trigger Shallow Thrust Fault Earthquakes. Science 306, 1164-1166.

Corral, A., 2004. Long-term clustering, scaling, and universality in the temporal occurrence of earthquakes. Phys. Rev. Lett. 92, 108501.

Crescentini, L., Amoruso, A., Scarpa, R., 1999. Constraints on slow earthquake dynamics from a swarm in central Italy. Science 286 (5447), 21322134 .

Davidsen, J., Stanchits, S., Dresen, G., 2007. Scaling and universality in rock fracture. Phys. Rev. Lett. 98, 125502.

Dieterich, J., 1994. A constitutive law for rate of earthquake production and its application to earthquake clustering. J. Geophys. Res. 99, 2601-2618.

Doubre, C., Peltzer, G., 2007. Fluid-controlled faulting process in the asal rift, djibouti, from 8 yr of radar interferometry observations. Geology $35(1), 69-72$.

Du, W.-x., Sykes, L. R., Shaw, B. E., Scholz, C. H., 2003. Triggered aseismic fault slip from nearby earthquakes, static or dynamic effect? J. Geophys. Res. 108, 2131.

Earle, P. S., Shearer, P. M., 1994. Characterization of global seismograms using an automatic-picking algorithm. Bull. Seis. Soc. Am. 84 (2), 366376.

Gao, H., Rice, J. R., 1986. Shear Stress Intensity Factors for a Planar Crack With Slightly Curved Front. J. Appl. Mech. 53, 774. 
Gao, H., Rice, J. R., Lee, J., 1991. Penetration of a quasi-statistically slipping crack into a seismogenic zone of heterogeneous fracture resistance. J. Geophys. Res. 96, 21535-21548.

Ghosh, A., Vidale, J. E., Sweet, J. R., Creager, K. C., Wech, A. G., Houston, H., Brodsky, E. E., 2010. Rapid, continuous streaking of tremor in Cascadia. Geochem., Geophy., Geosy. 11, 12010.

Grob, M., Schmittbuhl, J., Toussaint, R., Rivera, L., Santucci, S., Måløy, K. J., 2009. Quake catalogs from an optical monitoring of an interfacial crack propagation. Pure App. Geophys. 166, 777-799.

Hainzl, S., Scherbaum, F., Beauval, C., 2006. Estimating Background Activity Based on Interevent-Time Distribution. Bull. Seism. Soc. Am. 96, 313-320.

Helmstetter, A., Shaw, B. E., 2009. Afterslip and aftershocks in the rateand-state friction law. J. Geophys. Res. 114, B01308.

Hillers, G., Mai, P. M., Ben-Zion, Y., Ampuero, J.-P., 2007. Statistical properties of seismicity of fault zones at different evolutionary stages. Geophys. J. Int. 169, 515-533.

Hsu, Y.-J., Simons, M., Avouac, J.-P., Galetzka, J., Sieh, K., Chlieh, M., Natawidjaja, D., Prawirodirdjo, L., Bock, Y., 2006. Frictional Afterslip Following the 2005 Nias-Simeulue Earthquake, Sumatra. Science 312, 1921-1926.

Ito, Y., Obara, K., Shiomi, K., Sekine, S., Hirose, H., 2007. Slow Earthquakes Coincident with Episodic Tremors and Slow Slip Events. Science 315, 503-.

Kanamori, H., 1994. Mechanics of earthquakes. Annual Review of Earth and Planetary Sciences 22, 207-237.

Kaneko, Y., Avouac, J.-P., Lapusta, N., 2010. Towards inferring earthquake patterns from geodetic observations of interseismic coupling. Nature Geoscience 3, 363-369.

Kato, A., Obara, K., Igarashi, T., Tsuruoka, H., Nakagawa, S., Hirata, N., 2012. Propagation of slow slip leading up to the $2011 \mathrm{mw} 9.0$ Tohoku-Oki earthquake. Science 335 (6069), 705-708. 
La Rocca, M., Creager, K. C., Galluzzo, D., Malone, S., Vidale, J. E., Sweet, J. R., Wech, A. G., 2009. Cascadia Tremor Located Near Plate Interface Constrained by S Minus P Wave Times. Science 323, 620-

Lengliné, O., Marsan, D., 2009. Inferring the coseismic and postseismic stress changes caused by the $2004 M_{w}=6$ Parkfield earthquake from variations of recurrence times of microearthquakes. J. Geophys. Res. 114, B10303.

Lengliné, O., Schmittbuhl, J., Elkhoury, J., Ampuero, J. P., Toussaint, R., Måløy, K. J., 2011. Down-scaling of fracture energy during brittle creep experiments. J. Geophys. Res. 116.

Lengliné, O., Toussaint, R., Schmittbuhl, J., Elkhoury, J. E., Ampuero, J.P., Tallakstad, K. T., Santucci, S., Måløy, K. J., 2011. Average crack front velocity during subcritical fracture propagation in a heterogeneous medium. Phys. Rev. E. 84.

Linde, A. T., Gladwin, M. T., Johnston, M. J. S., Gwyther, R. L., Bilham, R. G., Sep. 1996. A slow earthquake sequence on the San Andreas fault. Nature 383, 65-68.

Liu, Y., Rice, J. R., Larson, K. M., 2007. Seismicity variations associated with aseismic transients in Guerrero, Mexico, 1995 2006. Earth Planet. Sci. Lett. 262, 493-504.

Lohman, R. B., McGuire, J. J., 2007. Earthquake swarms driven by aseismic creep in the Salton Trough, California. J. Geophys. Res. 112, B04405.

Måløy, K. J., Santucci, S., Schmittbuhl, J., Toussaint, R., 2006. Local waiting time fluctuations along a randomly pinned crack front. Phys. Rev. Lett. 96, 045501 .

Meade, B. J., 2007. Power-law distribution of fault slip-rates in southern California. Geophys. Res. Lett. 342, L23307.

Miyazaki, S., McGuire, J. J., Segall, P., 2011. Seismic and aseismic fault slip before and during the 2011 off the Pacific coast of Tohoku earthquake. Earth, Planets and Space 63, 637-642.

Molchan, G., 2005. Interevent Time Distribution in Seismicity: A Theoretical Approach. Pure Appl. Geophys. 162, 1135-1150. 
Nadeau, R. M., Dolenc, D., 2005. Nonvolcanic tremors deep beneath the San Andreas fault. Science 307 (5708), 389.

Nakatani, M., 2001. Conceptual and physical clarification of rate and state friction: Frictional sliding as a thermally activated rheology. J. Geophys. Res. 106, 13347-13380.

Obara, K., Hirose, H., Yamamizu, F., Kasahara, K., 2004. Episodic slow slip events accompanied by non-volcanic tremors in southwest Japan subduction zone. Geophys. Res. Lett. 31, L23602.

Perfettini, H., Avouac, J.-P., 2004. Postseismic relaxation driven by brittle creep: A possible mechanism to reconcile geodetic measurements and the decay rate of aftershocks, application to the Chi-Chi earthquake, Taiwan. J. Geophys. Res. 109, B02304.

Perfettini, H., Avouac, J.-P., Tavera, H., Kositsky, A., Nocquet, J.-M., Bondoux, F., Chlieh, M., Sladen, A., Audin, L., Farber, D. L., Soler, P., 2010. Seismic and aseismic slip on the Central Peru megathrust. Nature 465, 78-81.

Perfettini, H., Schmittbuhl, J., Cochard, A., 2003. Shear and normal load perturbations on a two-dimensional continuous fault: 1. Static triggering. J. Geophys. Res. 108, 2408.

Rice, J., Lapusta, N., Ranjith, K., 2001. Rate and state dependent friction and the stability of sliding between elastically deformable solids. Journal of Mechanics Physics of Solids 49, 1865-1898.

Rogers, G., Dragert, H., 2003. Episodic Tremor and Slip on the Cascadia Subduction Zone: The Chatter of Silent Slip. Science 300, 1942-1943.

Saichev, A., Sornette, D., 2007. Theory of earthquake recurrence times. J. Geophys. Res. 112, B04313.

Schmittbuhl, J., Delaplace, A., Måløy, K. J., Perfettini, H., Vilotte, J. P., 2003. Slow Crack Propagation and Slip Correlations. Pure and Applied Geophysics 160, 961-976.

Scholz, C. H., Wyss, M., Smith, S. W., 1969. Seismic and Aseismic Slip on the San Andreas Fault. J. Geophys. Res. 74, 2049. 
Segall, P., Desmarais, E. K., Shelly, D., Miklius, A., Cervelli, P., 2006. Earthquakes triggered by silent slip events on Kìlauea volcano, Hawaii. Nature $442,71-74$.

Shelly, D. R., 2010. Migrating tremors illuminate complex deformation beneath the seismogenic San Andreas fault. Nature 463, 648-652.

Shelly, D. R., Beroza, G. C., Ide, S., 2007. Complex evolution of transient slip derived from precise tremor locations in western Shikoku, Japan. Geochem., Geophy. Geosy. 8, 10014.

Shelly, D. R., Ellsworth, W. L., Ryberg, T., Haberland, C., Fuis, G. S., Murphy, J., Nadeau, R. M., Bürgmann, R., 2009. Precise location of San Andreas Fault tremors near Cholame, California using seismometer clusters: Slip on the deep extension of the fault? Geophys. Res. Lett. 36, L01303.

Takada, Y., Furuya, M., 2010. Aseismic slip during the 1996 earthquake swarm in and around the Onikobe geothermal area, NE Japan. Earth Planet. Sci. Lett. 290, 302-310.

Vidale, J. E., Shearer, P. M., May 2006. A survey of 71 earthquake bursts across southern California: Exploring the role of pore fluid pressure fluctuations and aseismic slip as drivers. J. Geophys. Res. 111, B05312.

Ward, I. M., Hadley, D. W., 1993. An introduction to the mechanical properties of solid polymers. Wiley.

Wech, A. G., Creager, K. C., Houston, H., Vidale, J. E., Nov. 2010. An earthquake-like magnitude-frequency distribution of slow slip in northern Cascadia. Geophys. Res. Lett. 372, L22310.

Wech, A. G., Creager, K. C., Melbourne, T. I., 2009. Seismic and geodetic constraints on Cascadia slow slip. J. Geophys. Res. 114, B10316. 
Figure 1: Left: Side view of the experimental setup. The bottom plate is separated from the upper one using a loading force applied by a rod connected to a stepping motor. The upper PMMA plate is attached to a stiff aluminum frame (short dashed lines). The load causes a deflection $u$ of the bottom plate and the propagation of an interfacial crack. The crack front is located at distance $\bar{a}$ from the loading point. The front advance is monitored by a high or slow speed camera set in vertical position, perpendicular to the crack plane. Right: detailed bottom view of the sample and the loading axis. The dark gray zone corresponds to the cracked zone. The multiple black areas show the acoustic sensors of the linear array.

Figure 2: Evolution of the loading force (red), and the loading displacement (blue) during an experiment. The crack is supposed to start moving at $t=45 \mathrm{~s}$ as evidenced by the force peak. The black box on the top figure represents a zoom displayed on the bottom figure. A transient variation of speed is superimposed to a constant loading displacement rate between $\mathrm{t}=65 \mathrm{~s}$ to $85 \mathrm{~s}$ (the black line corresponds to the average front position). Camera and acoustic time windows are displayed respectively as dark gray and light gray shaded areas.

Figure 3: A: Map of the local speeds of the front. Image scale is given by the length of the vector showing the front propagation direction which is $1.3 \mathrm{~mm}$ long. The front propagates from bottom to top. We observe small scale fluctuations of the crack front speed. Black dots represent hypocenters of optical events obtained after thresholding the velocity map and are displayed at the centroid of the corresponding high velocity cluster. B: Zoom on a subzone of the interface located in the black rectangle in A. C: Probability density functions (pdfs) of the local velocities computed for 6 experiments. We observe a power law decay of the pdfs for $v>\langle v\rangle$ with an exponent $\nu=-2.55$ compatible with Måløy et al. (2006).

Figure 4: Example of recorded signal of an acoustic event (AE). We observed a modification of the frequency content associated with the arrival of the AE wave train. The duration of the $\mathrm{AE}$ on the displayed channel is around $100 \mu \mathrm{s}$. Sampling rate is $5 \mathrm{MHz}$

Figure 5: Acoustic records of two channels during $100 \mathrm{~ms}$. We observe on these two channels an abundant activity as attested by the numerous peaks in the acoustic signals. Stars mark the identification of events after automatic processing of the two signals by a STA/LTA procedure and matching common detection. Rate of optical events recorded during the same time period is plotted in red. 
Figure 6: Distribution of normalized inter-event time for all experiments. Each experiment is represented by a different colour. The best gamma distribution fitting all the experiments, is represented by a black curve: $p(\tau)=C \tau^{\gamma-1} e^{-\tau / \beta}$, where $C=0.44, \beta=1.8$ and $\gamma=0.54$. Black filled diamonds show the inter-event time distribution computed from the ISC worldwide catalog for earthquakes with magnitude $\mathrm{M}>5.5$.

Figure 7: Evolution of the rate of $\mathrm{AE}$ (purple diamonds), of the average crack front speed (blue squares) and of the optical event (OE) rate (red circles) as a function of time. Rate are computed for interval of $0.2 \mathrm{~s}$ for a time period encompassing the loading transient shown in Figure 2. We observe that the rates of $\mathrm{AE}$ and $\mathrm{OE}$ are well correlated with the variation of the crack front speed at this broad time scale.

Figure 8: Top: Crack front speed as a function of time and position along front during one experiment. High velocities appear as streaks that extend along the crack front direction. Bottom left: zoom that corresponds to the space time domain delimited by the black rectangle in the upper figure. White dashed lines show the migration during two high velocity episodes. Slope of these lines gives an estimated migration speed of the order of $2 \mathrm{~cm} / \mathrm{s}$.

Figure 9: Cross-correlation function $C(\Delta t)$ between the rate of acoustic events (AE) and the rate of optical events (OE). AE rate and $\mathrm{OE}$ rate are computed as the number of events during intervals of $5 \mathrm{~ms}$ and mean is remove from the time-series. The crosscorrelation function corresponds to an averaged function computed over 6 experiments. We observe that the maximum of the correlation function is found at zero time lag. We also notice the increase of the correlation function around the peak, suggesting that $\mathrm{OE}$ are clustered in time for some duration before and after an AE. The inset figure shows the normalized autocorrelation functions for the AE (black curve) and the OE (gray curve). Both functions show some increase around zero time lag supporting the interplay between these two modes of deformation. 


\section{*Highlights}

$>$ We build an original experiment designated to study the relation between slow and dynamic deformations in the brittle creep regime.

$>$ We observe numerous acoustic events in relations with local creeping episodes.

$>$ In relation with faulting processes, we propose that earthquake swarms are driven by local slow slips. 


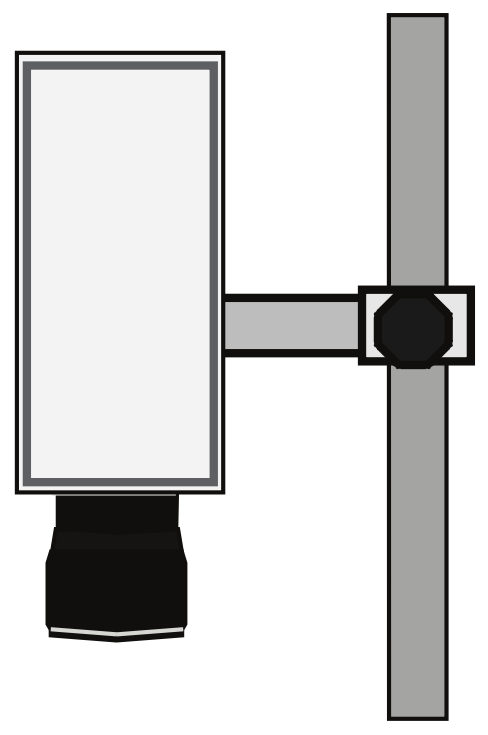
stage and stepping motor

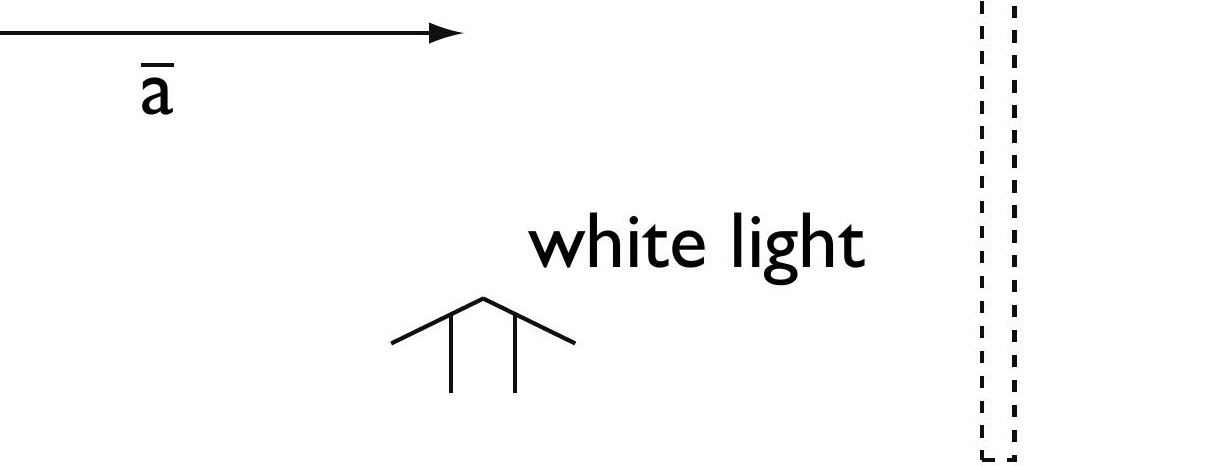


Click here to download Figure: fig2.eps
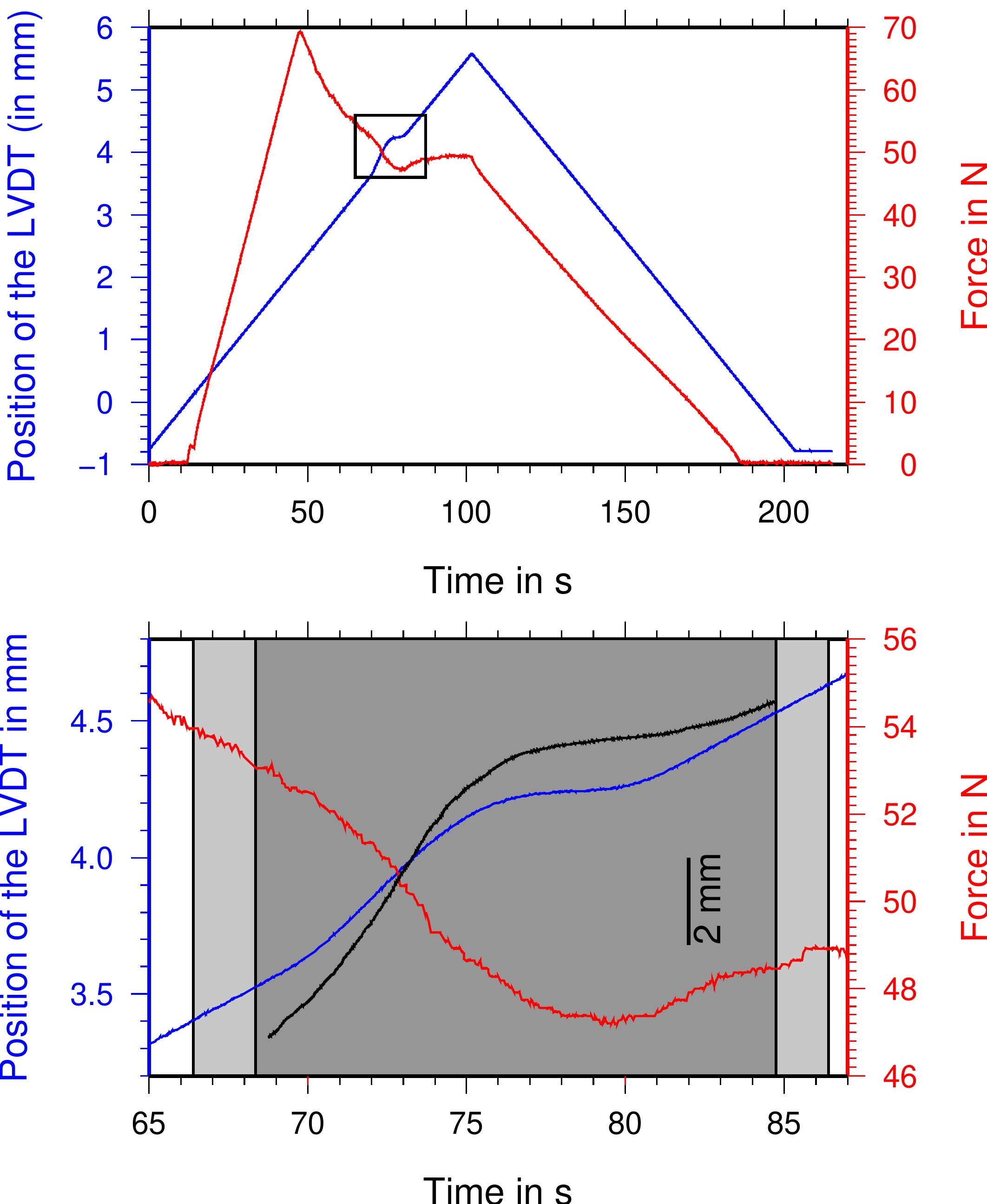
Figure 3

Click here to download Figure: fig3.eps
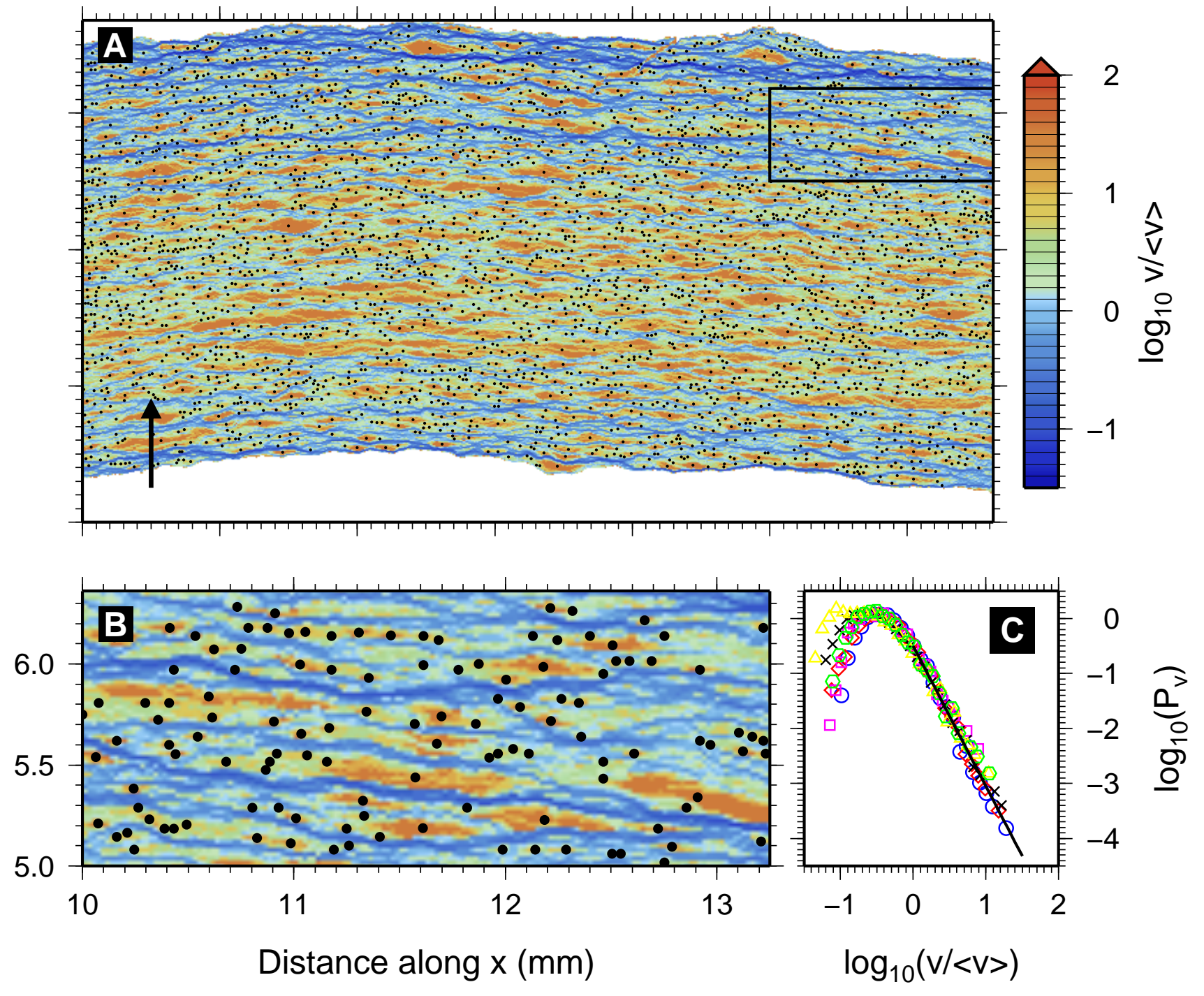
Figure 4
Click here to download Figure: fig4.eps

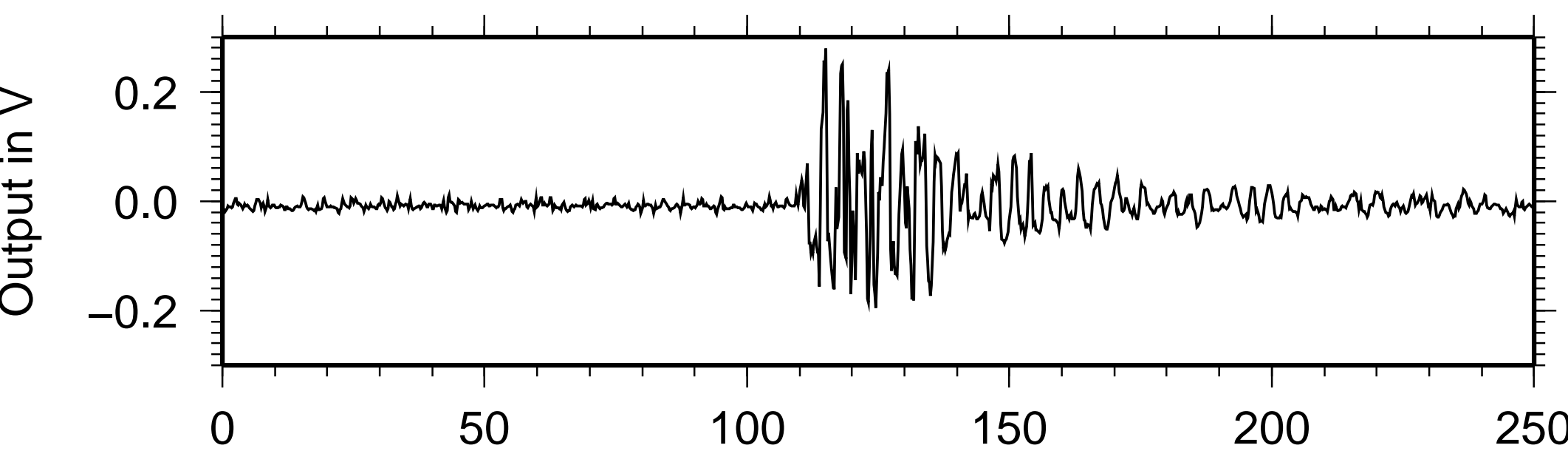

Time in $\mu \mathrm{s}$ 
Click here to download Figure: fig5.eps

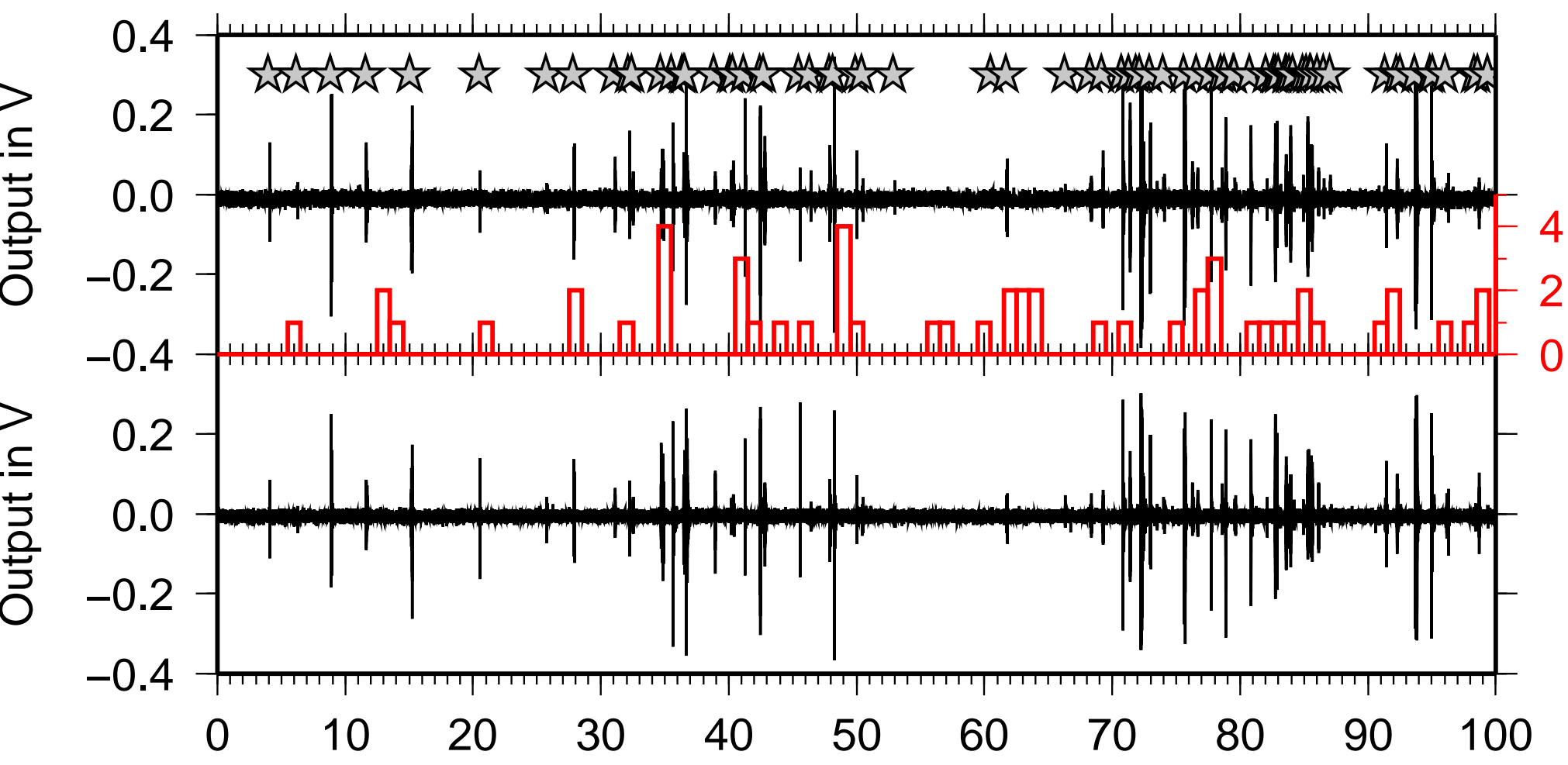

Time in $\mathrm{ms}$ 
Figure 6
Click here to download Figure: fig6.eps

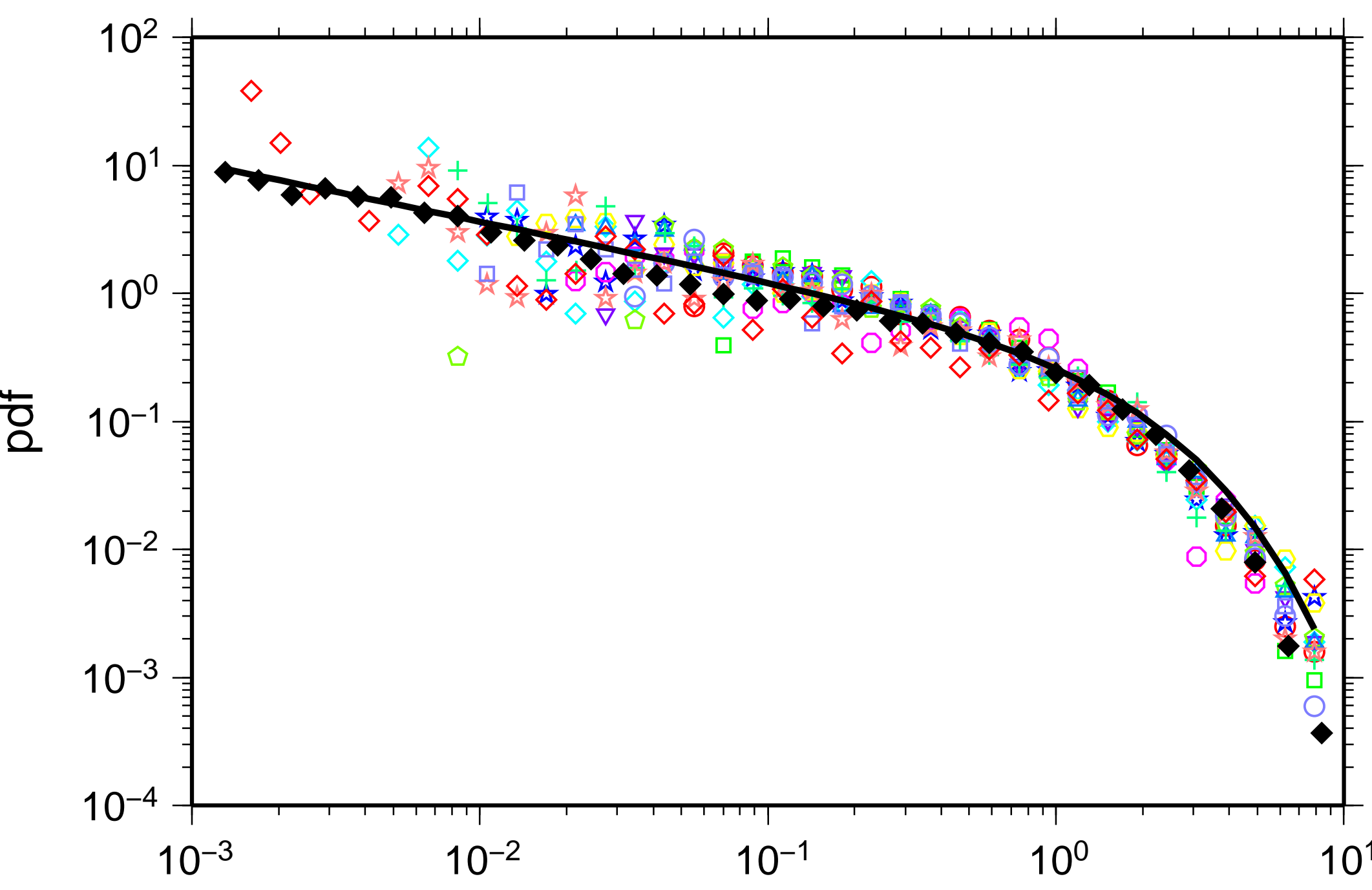

Normalized inter-event time 
Click here to download Figure: fig7.eps

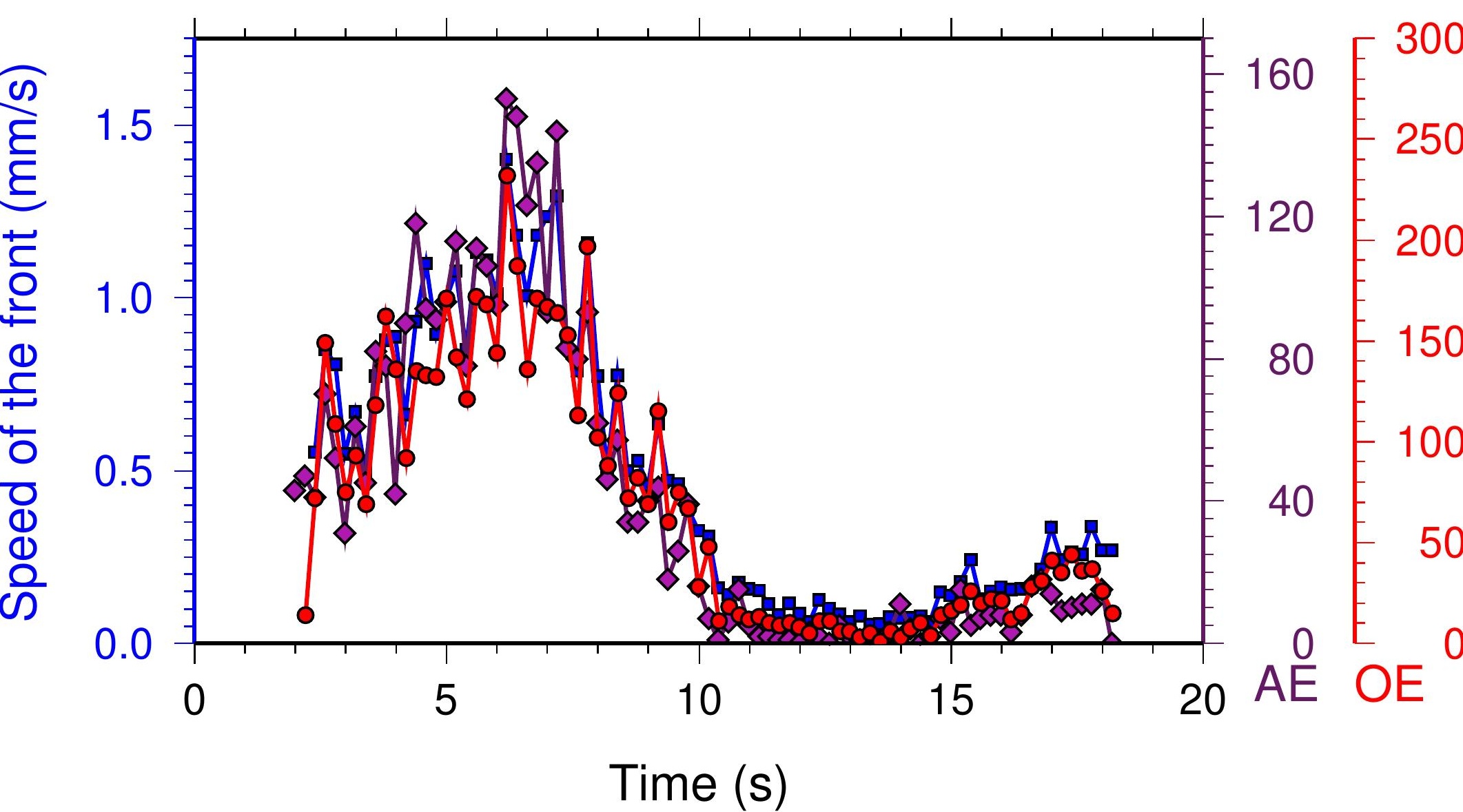




\section{Distance along front (mm)}

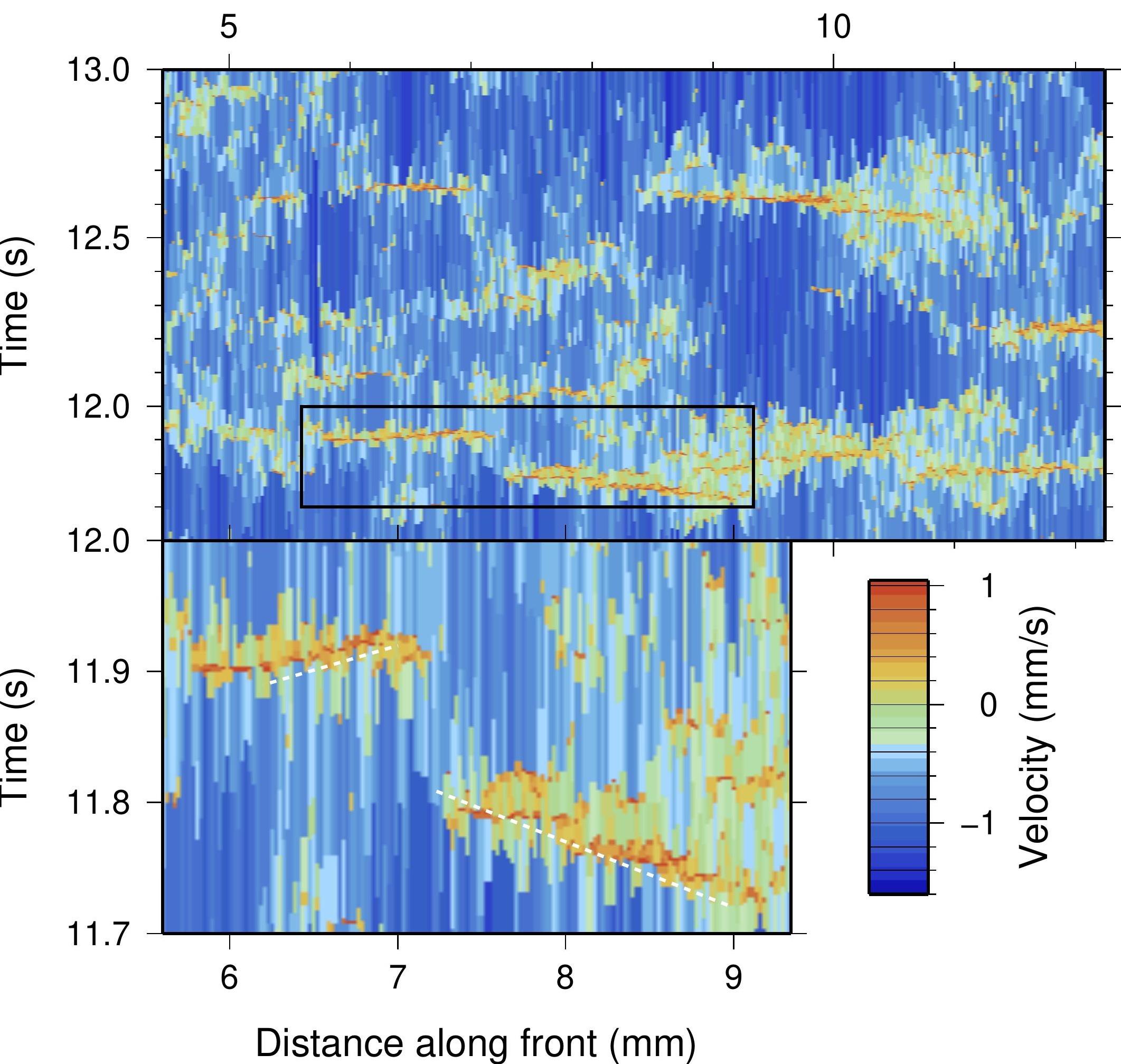




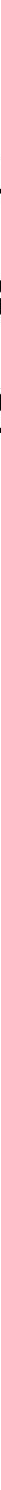

Time delay (in s) 
Supplementary material (Table I)
Click here to download Supplementary material for on-line publication only: table_SM.pdf

(n)

$\sqrt{2}$

(1)

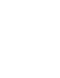

(1)

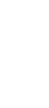

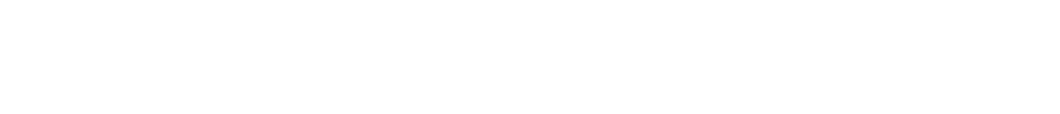

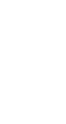
(1) $\sqrt{20}$

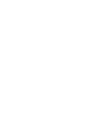

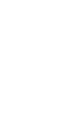

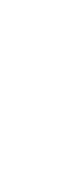
( . . . .

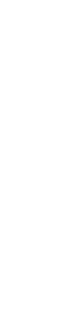

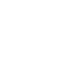

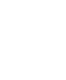
.

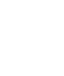
.

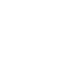
.

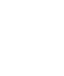
.

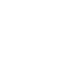
. 